\title{
Barriers to Provision of Spiritual Care in Mental Health Care Centers: A Qualitative Content Analysis
}

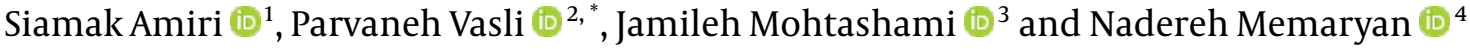 \\ ${ }^{1}$ Student Research Committee, School of Nursing and Midwifery, Shahid Beheshti University of Medical Sciences, Tehran, Iran \\ ${ }^{2}$ School of Nursing and Midwifery, Shahid Beheshti University of Medical Sciences, Tehran, Iran \\ ${ }^{3}$ Shahid Beheshti University of Medical Sciences, Tehran, Iran \\ ${ }^{4}$ Mental Health Department, Spiritual Health Research Center, School of Behavioral Sciences and Mental Health (Tehran Institute of Psychiatry), Iran University of Medical \\ Sciences, Tehran, Iran \\ "Corresponding author: School of Nursing and Midwifery, Shahid Beheshti University of Medical Sciences, Tehran, Iran. Email: p-vasli@sbmu.ac.ir
}

Received 2021 May 03; Revised 2021 August 10; Accepted 2021 September 15.

\begin{abstract}
Background: Spiritual care (SC) is an appropriate source for adaptation and coping with disease and life stressors. Although SC contributes to the quality of life and mental health of clients, there are barriers to the provision of such care.

Objectives: This study aimed to explore barriers to the provision of SC in mental health care centers from the viewpoints of mental health care provider (MHCP) experts.

Methods: This qualitative study was conducted based on conventional content analysis on 19 participants who were selected from different groups of MHCPs. The participants were selected by purposive sampling method from December 2019 to December 2020 in Karaj and Tehran, Iran, by considering the maximum diversity. Data were collected through semi-structured interviews with participants during an average time of 60 min per interview until data saturation. The transcripts of the interviews were analyzed by qualitative content analysis.

Results: Barriers to SC were obtained in four categories and 10 subcategories. The categories included "SC concept-related barriers", "barriers related to MHCPs", "organizational barriers", and "barriers related to SC recipients."

Conclusions: This study demonstrated various barriers to the provision of SC to the clients of health care centers that should be removed to promote the level and quality of SC.
\end{abstract}

Keywords: Barriers, Health Care Providers, Mental Health, Qualitative Content Analysis, Spiritual Care

\section{Background}

Spirituality, as one of the important aspects of a holistic approach to health care, can lead to the desired improvement of the client's health (1). This concept is defined as a dynamic and intrinsic aspect of humanity through which people seek the meaning, objective, and ultimate transcendence and experience a relationship with themselves, family, others, society, community, nature, and important or sacred things (2). Spiritual care (SC) is a subjective and dynamic concept that reflects the uniqueness of care and integrates all other aspects of care (3). Besides, SC is a proper source for adaptation and coping with disease and life stressors. This concept contributes to the promotion of the quality of life and mental health of patients (4$6)$. The provision of SC to people with mental health problems enhances their hope, mitigates depression, and reduces suicide attempts (7).

While the majority of health professionals believe that paying attention to the patient's spiritual beliefs and provision of spiritual support can have a positive effect on patients $(8,9)$, it is often ignored by them $(10,11)$. This shortcoming also exists in Iran as a Muslim country where there should be the opportunity to present SC. Although the historical role of spirituality and religion has been recognized as a key component of treatment and support by some Iranian historical scientists (e.g., Avicenna and Zakariya alRazi), recent studies indicate a low provision level of this type of care in Iran (12). Some barriers to the provision of SC hinder meeting the needs of patients (13).

Despite available studies on barriers to SC in various areas of health care with participants from different members of health care providers (HCPs), there are few studies on barriers to SC in terms of mental health. For instance, a study in the USA examined the barriers to SC provision among psychiatric nurses. According to this study, the most common barriers in this regard were the concerns of psychiatric nurses about stimulating or reinforc-

Copyright (c) 2021, Author(s). This is an open-access article distributed under the terms of the Creative Commons Attribution-NonCommercial 4.0 International License (http://creativecommons.org/licenses/by-nc/4.0/) which permits copy and redistribute the material just in noncommercial usages, provided the original work is properly cited. 
ing distorted religious or psychotic thought of patients following SC provision, lack of knowledge, and lack of time (14). Other studies examined SC-related barriers to health care, specifically in a special group of HCPs. In Iran, a study on the experiences of oncology nurses about barriers and facilitators affecting SC provision revealed that attitudinal-behavioral restriction (e.g., insensitivity to SC) and uncoordinated environments (e.g., lack of funding and the use of inexperienced people) could prevent SC provision (15). According to a cross-sectional study in Jordan, nurses believed that the most common barriers to providing patients with SC were lack of space for SC provision, no access to a clergyman, insufficient time, insufficient skills and competencies, lack of facilities, and religious/spiritual resources in the hospital, and insufficient knowledge (16). In another study, the lack of training, concern, or the experience of fear of expressing spirituality as a taboo were the most important barriers to the practical integration of spirituality/religion in viewpoints of American social workers (17). In a qualitative study in Australia and New Zealand, oncology physicians and incurable patients reported barriers such as the interference of spirituality concept with religion, the spirituality level of the presenter, and organizational factors (e.g., inaccessibility to chaplain), and historical factors (e.g., separation of science and spirituality) in medical education (18).

Despite the importance of providing SC to people with mental health problems, there is no study on the barriers to the provision of this type of care in Iran.

\section{Objectives}

The present study aimed to fill the existing knowledge gap by exploring the barriers to SC provision in mental health service providers from the viewpoints of different groups of mental health care providers (MHCPs). Overall, the qualitative study may pave the road for understanding the experience of people and the meaning of their experienced events in the context of community culture (19).

\section{Methods}

\subsection{Study Design}

This qualitative study was performed using conventional content analysis. The data needed for this purpose were collected using in-depth interviews with the participants, without any previous hypotheses (20).

\subsection{Research Setting and Participants}

This study was conducted in the psychiatric inpatient wards of teaching hospitals, counseling clinics, and outpatient psychological services in Tehran and Karaj, two large Iranian metropolises. To achieve maximum diversity in sampling, participants from different groups of MHCPs and SC were included in the study by purposive sampling to better identify barriers to providing SC. Participants were selected from those who had the most experience. Some participants were also recruited to study by initial participants. The participants were selected among psychiatrists, psychologists, psychiatric nurses, and religious counselors of different ages, genders, and work experiences.

Inclusion criteria were at least three years of work experience as MHCPs or having SC-related studies, and being a Muslim. There were a total of 19 participants, including six nurses, six clinical psychologists, five psychiatrists, and two clergymen. The participants were in the age range of 31 - 68 years (mean 47.68 years) (Table 1 ).

\subsection{Data Collection}

Data were collected through semi-structured and indepth interviews from December 2019 to December 2020. The interviews were conducted by the first author, a psychiatric nurse with more than 15 years of experience and research experience in spirituality and mental health. Sampling continued until data saturation, i.e., until no new data were obtained. Each interview lasted 60 min on average. The time of the interviews was suggested by the participants, and they were often done at midday when they had free time. Interviews were often conducted in the education department of the hospital or the clinic management room in a quiet environment away from environmental stimuli. Three participants (No. 3, 9, and 14) were interviewed twice. The opening questions included, "What do you do when you encounter the spiritual needs of patients? Based on your experiences, what are the barriers to responding to the client's spiritual needs? These were followed by exploratory and follow-up questions for in-depth interviews, including "What are factors affecting your care delivery? And Can you explain more?" The interviews were recorded and then transcribed verbatim.

\subsection{Analysis of Data}

Data analysis started simultaneously with data collection using the conventional qualitative content analysis proposed by Graneheim and Lundman. The first author was responsible for data analysis under the guidance of the corresponding author. Before coding, the transcribed text was read several times to have a general overview of the text. After that, the meaning units in each transcript were identified, and the initial codes were assigned. Descriptive and in-vivo coding was used for initial analysis. The various codes were compared based on differences and similarities 


\begin{tabular}{|c|c|c|c|c|c|c|}
\hline No. & Age & Sex & Type of Participant & Degree & Position & $\operatorname{Experience}(\mathbf{y})$ \\
\hline $\mathbf{1}$ & 36 & Female & Psychologist & MA & Therapist & 9 \\
\hline 2 & 31 & Female & Psychologist & MA & Therapist & 6 \\
\hline 3 & 36 & Female & Psychologist & MA & Therapist & 8 \\
\hline 4 & 45 & Female & Psychologist & PhD, Assistant Professor & Therapist, Researcher & 12 \\
\hline 5 & 55 & Female & Psychologist & PhD, Associate Professor & Therapist, Researcher & 23 \\
\hline 6 & 59 & Male & Psychologist & PhD, Professor & Therapist, Researcher, Theoretician & 32 \\
\hline 7 & 41 & Male & Psychiatric nurse & MS & Nurse & 15 \\
\hline 8 & 46 & Female & Nurse Scholar & PhD, Assistant Professor & Nurse, Researcher & 7 \\
\hline 9 & 57 & Male & Nurse Scholar & PhD, Professor & Researcher, Manager & 27 \\
\hline 10 & 41 & Female & Psychiatric nurse & MS & Nurse, Researcher & 16 \\
\hline 11 & 43 & Female & Psychiatric nurse & MS & Nurse & 17 \\
\hline 12 & 36 & Male & Nurse & BSN & Nurse & 9 \\
\hline 13 & 49 & Male & Psychiatrist & MD, Assistant Professor & Therapist, Researcher, Manager & 15 \\
\hline 14 & 48 & Male & Psychiatrist & MD, Assistant Professor & Therapist, Researcher & 12 \\
\hline 15 & 63 & Male & Psychiatrist & MD, Associate Professor & Therapist, Researcher, Manager & 28 \\
\hline 16 & 68 & Male & Psychiatrist & MD, Professor & Therapist, Researcher, Manager & 31 \\
\hline 17 & 47 & Female & Psychiatrist & MD, Assistant Professor & Therapist, Researcher & 9 \\
\hline 18 & 53 & Male & Religious/spiritual counselor & Clergyman, Assistant Professor & Counselor, Researcher & 21 \\
\hline 19 & 52 & Male & Religious/spiritual counselor & Clergyman, Assistant Professor & Counselor, Researcher, Manager & 19 \\
\hline
\end{tabular}

and sorted into 10 tentative subcategories. Afterward, similar subcategories were grouped into four categories. During the analysis, categories, subcategories, and codes were continuously compared with each other. Disagreements between researchers during a process of reflection and discussion led to agreement on how to sort the code. Data were analyzed using MAXQDA version 10 software.

\subsection{Data Trustworthiness}

In this study, the credibility, dependability, transferability, confirmability, and authenticity criteria were used for the scientific accuracy and validity of data. In all interviews, the relationships between the objective, questions, and participants' answers were reviewed and confirmed by the research team. We tried to increase the trustworthiness of data by long-time immersing in data and starting interviews based on the required data. Coding and data analysis were carried out through peer review by two researchers simultaneously. This was followed by reviewing the transcripts of the interviews and initial codes by three participants (member check) to increase the dependability of data. Other procedures for enhancing the trustworthiness of the study included the preparation of accurate reports of the study method, including participants' characteristics, sampling method, data collection method, re- search setting, and expression of results along with participants' quotations $(20,21)$.

\section{Results}

A total of 748 codes were extracted. According to the analysis of participants' answers, the SC barriers were classified into four categories of "SC concept-related barriers", "barriers related to MHCPs", “organizational barriers", and "barriers related to SC recipients" (Box 1), each of which is described in detail in the following.

\subsection{SC Concept-Related Barriers}

One of the barriers that can be considered as the cause of other barriers is the characteristics of the concept of SC, including "overlap and diversity of spiritual and religious approaches" and "ambiguity in the meaning and methods of SC". These two features caused difficulty in SC provision, and thus, HCPs refused to provide it.

\subsubsection{Overlap and Diversity of Spiritual and Religious Ap- proaches}

Participants stated the overlap between spirituality and religion, as well as the diversity of spiritual and religious perspectives and approaches, as fundamental barri- 


\begin{tabular}{|l}
\hline Box 1. Spiritual Care Barriers in Mental Health Care Settings \\
\hline Main Categories and Subcategories \\
\hline SC concept-related barriers \\
Overlap and diversity of spiritual and religious approaches \\
Ambiguity in the meaning and methods of SC \\
\hline Barriers related to MHCPs \\
\hline Weakness in self-awareness \\
\hline Lack of support for spirituality \\
Insufficient competence \\
\hline Organizational barriers \\
\hline Non-priority of SC \\
Lack of resources \\
\hline Lack of culturally tailored guidelines \\
\hline Barriers related to SC recipients \\
\hline Intrapersonal barriers \\
\hline Interpersonal barriers \\
\hline
\end{tabular}

ers to SC provision. Regarding the overlap of spirituality and religion as a barrier, participant No. 9 explained:

"When we see spirituality equal to religion, some people whose religious beliefs are not like those of the general public are pushed to the margin; these groups will be certainly damaged because we present our activities to the average community. We have to draw a line between spirituality and religion. I mean, we should define correctly religion and spirituality. We have to clarify their interactions and differences with each other. Unfortunately, this border is not clear yet." (A nurse scholar)

\subsubsection{Ambiguity in the Meaning and Methods of SC}

Participants believed that ambiguity in the meaning of the concept of SC, as well as in its provision methods, is among the factors of their confusion. They pointed out that this ambiguity was also found in the content of SC. For example, a psychiatrist commented on the ambiguity of the concept as follows:

"We mainly face a vague issue as we have some completely secular therapists in psychotherapy who claim to have a spiritual approach than those who are fundamentally religious. They all have something in common, so we need to know what we are talking about?" (Participant No. 13)

\subsection{Barriers Related to MHCPs}

One of the categories extracted from the participants' answers was the barriers related to MHCPs. Data analysis revealed that "poor self-awareness", "lack of support for SC", and "insufficient competence for SC" were among the barriers to the provision of appropriate SC by MHCPs from the viewpoints of some participants.

\subsubsection{Weakness in Self-awareness}

According to the participants' views, their lack of analysis of their behaviors and poor self-awareness, as well as their unresolved spiritual distress, are factors that prevent SC provision. About the spiritual distress, participant No. 2, a clinical psychologist, stated that:

"I have not yet resolved my spiritual distress. We also have conflicts that have not been resolved for ourselves. We do not know the meaning of our own lives and doubt our spiritual beliefs; this is itself a barrier."

\subsubsection{Lack of Support for Spirituality}

Another subcategory of barriers related to MHCPs was the lack of professionals' support for SC, which included dealing with spirituality from a positivist perspective, considering spirituality as pseudoscience, believing that spiritual problems are caused by mental disorders, and insignificant consideration of clients' spiritual needs. The following are two quotes from this subcategory:

"An important part of the academic opposition to spirituality is that they judge spirituality from a positivist perspective. From this viewpoint, when you say that prayer as a spiritual act causes lower stress, spirituality is not different from activities like exercise. This is because they show that just as prayer can bring relaxation, exercise can do the same and may even have a greater effect than prayer, while we cannot test all aspects of spirituality with experimental tools." (Participant No. 14, a psychiatrist)

"Some of our colleagues believe that the spiritual issues raised by patients to be rooted in illness and mental disorders and say that spiritual struggles also subside with medication and treatment of mental disorders ... In fact, it is right that this person is not depressed now by taking medication, but the meaning of his/her life is not yet solved by medicine ..." (Participant No. 18, a religious/spiritual counselor)

\subsubsection{Insufficient Competence}

Almost all participants pointed out the inadequate competence of MHCPs in SC provision as a deficiency in assessing spiritual needs, communicating with the client, answering the client's spiritual questions and concerns, implementing spiritual interventions, and various spiritual intervention approaches. Concerning the inadequate competence of MHCPs in assessing clients' spiritual needs, participant No. 3 said:

"Clients themselves do not clearly ask for their spiritual issues, and it is the therapist who has to look into them. Now, I do not know what questions I should use to 
assess the patient's spirituality; that's why spirituality is often overlooked." (A psychologist)

Another participant also commented on the lack of knowledge about SC approaches as follows:

"Spiritual interventions have different methods such as yoga, self-awareness, religion-based or spiritually integrated psychotherapy. Nurses, psychiatrists, and psychologists are not very familiar with these and often use the way they know, not the way that suits the patient." (Participant No. 4, a psychologist)

\subsection{Organizational Barriers}

Participants believed that some barriers to SC provision were related to organizations. They mentioned the shortage of resources, the non-priority of SC in organizational planning, and the absence of a culturally appropriate SC guideline to be among these barriers.

\subsubsection{Non-priority of SC}

Some of the participants suggested the non-priority of SC provision in the relevant institution. These included lack of an action plan for SC implementation, lack of indicators for SC provision in accreditation and quality improvement, the absence of tariffs for SC providers, and paying less attention to the educational needs of HCPs in terms of SC. About the lack of SC provision criteria in accreditation and quality improvement, a nurse participant commented:

"There is no place at all in the quality care delivery system to offer spiritual care to patients, and the indicators of this care are not clear. I, as a nurse, consider this as an unnecessary task that I may do if I have the time to do." (Participant No. 10)

Another participant noted the lack of an action plan to implement SC:

"The management of an organization must devote part of its action plan to the promotion of spiritual care. We have almost no special spiritual care program in any center." (Participant No. 8, a nurse scholar)

\subsubsection{Lack of Resources}

Most of the participants mentioned the lack of resources, such as lack of resource allocation, workforce shortage, and lack of physical space necessary to provide $\mathrm{SC}$ and perform the religious duties of the clients as barriers to SC. Regarding the lack of workforce, participant No. 12 explained:

"Another factor is that we do not have enough time for this care, as we are in nursing shortage. Possibly a nurse would like to teach others a few things he/she has learned in this field, but he/she gets so tired in the ward and so tangled in the routine task that is exhausted." (A nurse)
About the lack of physical space to provide SC, one of the participants stated that:

"Spiritual intervention does not work with one or two sessions, and these people need regular follow-ups. Unluckily, there is no physical space to work in hospitals and medical centers... The patient who comes for a visit has to wait until the afternoon. As lots of patients arrive at the hospital early in the morning, they can no longer wait until the evening." (Participant No. 1, a psychologist)

\subsubsection{Lack of Culturally Tailored Guidelines}

The last subcategory in organizational barriers was the lack of culturally tailored guidelines. Participants also noted the hazy scope of responsibilities and job descriptions of different groups of MHCPs in SC provision. The following is one quotes from participants:

"We have not yet been able to make a clear framework for spiritual interventions in mental health. No guideline and protocol are matching our state and our patients." (Participant No. 15, a psychiatrist)

\subsection{Barriers Related to SC Recipients}

The fourth category is barriers related to SC recipients that do not directly hinder SC implementation but indirectly affect SC provision. This finding was obtained based on two subcategories.

\subsubsection{Intrapersonal Barriers}

Some participants mentioned some barriers of intrapersonal origin related to $\mathrm{SC}$ recipients, including the severity of mental disorders, nihilistic beliefs of SC recipients, and their opposition to spirituality because of attributing problems to religion and God. The following is one quotes from this subcategory:

"One of the challenges we have with some clients is that they experience a sense of futility because they see the world too empty. They have no vision of the future, do not believe in the afterlife at all, and have no spiritual experience. I think spirituality interventions cannot help these people, even though they have a lot of problems about it." (Participant No. 5, a psychologist)

\subsubsection{Interpersonal Barriers}

Some participants indicated barriers of interpersonal origin related to SC recipients, including experiencing moral injury and poor socioeconomic status of clients. In this regard, one participant noted that:

"People who have experienced multiple moral injuries and have changed their attitudes toward people, the world, and even religious people hardly respond to spiritual interventions. These people have deep spiritual struggles." (Participant No. 17, a psychiatrist) 


\section{Discussion}

This qualitative study aimed at explaining and describing the barriers to SC provision to clients of mental health care centers. The results demonstrated that SC provision barriers fall into four main categories: (1) SC conceptrelated barriers, (2) barriers related to MHCPs, (3) organizational barriers, and (4) barriers related to SC recipients.

One of the main barriers to SC provision mentioned by most of the participants was those related to the concept of SC. The overlap and diversity of spiritual and religious approaches, on the one hand, and the ambiguity in the definition and method of SC provision, on the other hand, are the two factors that obscure the concept of SC and restrict its implementation. In line with this finding, Hvidt et al. studied the identification, organization, and prioritization of SC experiences and perceptions in researchers, students, and clinical therapists. They found that an unclear definition of SC was a reason for the low implementation of this care (22). Likewise, Holmes found that ambiguity in the definitions of spirituality and religion and the multiplicity of SC provision models confused SC providers in the hospital from the perspectives of managers, policymakers, researchers, and professors (23).

The ambiguity in defining spirituality as a complex and multifaceted concept seems to be a major challenge. Although spirituality and religion are associated historically with medical issues, researchers have failed to provide a single definition for them (24), which is a barrier to SC. In this respect, it is difficult to define and study spirituality and find practical models to integrate it into health care in Iran as a country with an Islamic context (25). However, the use of spirituality in health services requires understanding and defining it precisely and determining its relationship with health and wellness $(26,27)$.

The second finding was barriers related to poor selfawareness of MHCPs in SC provision. Poor self-awareness and unresolved spiritual distress of SC providers are the other barriers to SC provision. Consistent with these results, Bar-Sela et al. found that self-awareness and one's communication with personal spirituality were the keys factor influencing SC provision (28). Therefore, HCPs can practice and improve their spiritual self-awareness and awareness of their fears and lack of knowledge to increase their spirituality and become sensitive to the spiritual needs of clients and their families and integrate SC into clinical care $(29,30)$.

Another aspect of the barriers related to MHCPs was the lack of support for spirituality. Koren and Papamiditriou also stated that support and a positive attitude toward spirituality were important factors in SC provision (31). Another study found that physicians and nurses who described themselves as less spiritual were less likely to participate in spirituality training courses (32). Although
HCPs were found to have a positive attitude toward SC due to the existence of religious foundations in the heart of Iranian society, they do not support it in practice (33). The reason for the lack of support for SC from our participants may be related to another aspect of barriers related to MHCPs, namely inadequate competence. In Iran, Zakaria Kiaei et al. revealed low competence in SC among the important barriers to providing this type of care (12). Musa et al. and Bar-Sela et al. conducted a cross-sectional study on Egyptian nurses and a study on physicians and nurses in Central Asian countries, respectively. According to their results, lack of education, knowledge, and competence included some barriers to SC provision $(16,28)$. The authors believe that a reason for the inadequate competence of Iranian MHCPs in SC provision may be the lack of standard and adequate education in educational courses or in-service education thereafter and low motivation toward their specialty profession.

The third category of the findings was the organizational barriers as non-priority of SC and lack of resources. Selman et al. conducted a qualitative study examining the experiences and research priorities in an international sample of patients with life-limiting disease and their caregivers in nine countries. They found that the non-priority of SC was one of the barriers to its implementation (2). Holmes studied the viewpoints of stakeholders (i.e., managers, policymakers, researchers, and professors) on the role of SC in Australian hospitals and mentioned the lack of resources, budgets, and priorities of decision-makers in the organization as barriers to SC implementation (23). In a qualitative study, Holyoke and Stephenson confirmed the importance of organizational support for SC implementation and reported that SC required at least nine organizational principles and practices (34).

Another dimension of organizational barriers to SC provision is the unavailability of culturally tailored guidelines. A qualitative study by Narayanasamy and Owens on determining the spiritual needs of patients and nurses' responses revealed the ambiguous definition of the concept of spirituality and the role of nurses concerning SC. They also observed that nurses respond to it with different approaches, the SC approach was largely unsystematic and provided accidentally in a personal and intuitive manner (35). Rushton also considers the lack of guidelines among the barriers to the use of SC by nurses (36). Lack of guidelines, the unclear scope of caregivers' duties, and lack of role transparency in various mental health disciplines are barriers to providing SC (37).

The last category of findings is barriers related to SC recipients in the form of barriers with intrapersonal and interpersonal origins. Regarding the interpersonal barriers for SC recipients, a qualitative study and interview with psychologists indicated that one of the barriers to us- 
ing spirituality and religion in treatment was client resistance as the desired treatment by a fellow therapist, feelings of guilt in seeking treatment based on religious beliefs, or passivity in making decisions for treatment (38). In another qualitative study in Taiwan, physicians and nurses stated that the patient's emotional, physical, and social conditions, especially impaired consciousness, were among the major challenges to effective SC provision to patients (39). Contrary to this finding of our study, another study revealed that clients with acute conditions, especially depression, were more inclined to spiritual interventions (40).

Altogether, this study is valuable in terms of some aspects. First, given the importance of SC in mental disorders, a few studies on spirituality in mental health have examined the barriers to SC provision from the viewpoints of MHCPs. Second, the use of a qualitative approach in this study could reveal more deeply the participants' experiences about SC provision to the clients of mental health centers. The third one is the participation of various health care professionals in the study. As denoted in articles with multidisciplinary authorship or those in which interviewees were participants from different professional groups, SC should be implemented by several members of the health care team (41).

\subsection{Conclusions}

The findings of this study demonstrated that four categories of barriers (i.e., "SC concept-related barriers", "barriers related to MHCPs", "organizational barriers", and "barriers related to SC recipients") disrupt the provision of SC to clients of health care centers. Our findings highlight the need for a multilevel and comprehensive approach to address the multiple barriers to SC provision in the health care system. The authors believe that the most important barriers to SC provision in mental health care centers are ambiguity in understanding the concept of SC and the use of different approaches in SC provision. Therefore, it is suggested that a clearer definition of SC be provided based on the spiritual needs of patients receiving health services, considering the predominant cultural and religious context (Islam) in Iran. A culturally tailored guideline with qualitative, quantitative, and Delphi studies, with the consensus of experts, should be prepared for the coherent provision of SC. It is also suggested to pay special attention to developing upstream policies, allocating the required resources for SC, and developing indicators to evaluate the use of guidelines in the accreditation process of the health care system. The SC courses should also be included in the curriculum to the universities, and MHCPs should be trained with ongoing education. In this way, MHCPs will achieve adequate competence in the assessment of and responding to the spiritual needs of their clients. Although this study suffers from limitations due to its implementation in a specific cultural and religious context, it can be generalized to and used in similar communities and contexts.

\section{Acknowledgments}

The authors appreciate all the participants in the study.

\section{Footnotes}

Authors' Contribution: SA and PV did the planning and design of the study, performed analysis, and wrote the manuscript. SA conducted the interviews. This study was supervised by PV. JM and NM were involved in controlling interviews and data analysis. All authors were in close collaboration and responsible for critical revisions of the manuscript. All authors read and approved the final manuscript.

Conflict of Interests: The authors state that there is no conflict of interest in the present study.

Ethical Approval: This study was extracted from a doctoral dissertation registered with the ethics code IR.SBMU.PHARMACY.REC.1398.121 in the Research Ethics Committee of Shahid Beheshti University of Medical Sciences.

Funding/Support: This study did not receive any financial support and funding from organizations or institutions.

Informed Consent: Written informed consent was obtained from the participants, and they were reassured about the confidentiality of their answers and the right to withdraw at any stage of the study.

\section{References}

1. Carey LB, Mathisen BA, Koenig H. Spiritual care for allied health practice: A person-centered approach. Jessica Kingsley Publishers; 2018. p. 11-20.

2. Selman LE, Brighton LJ, Sinclair S, Karvinen I, Egan R, Speck P, et al. Patients' and caregivers' needs, experiences, preferences and research priorities in spiritual care: A focus group study across nine countries. Palliat Med.2018;32(1):216-30. doi:10.1177/0269216317734954.[PubMed: 29020846]. [PubMed Central: PMC5758929].

3. Ramezani M, Ahmadi F, Mohammadi E, Kazemnejad A. Spiritual care in nursing: A concept analysis. Int Nurs Rev. 2014;61(2):211-9. doi: 10.1111/inr.12099. [PubMed: 24712404].

4. Counted V, Possamai A, Meade T. Relational spirituality and quality of life 2007 to 2017: An integrative research review. Health Qual Life Outcomes. 2018;16(1):75. doi: 10.1186/s12955-018-0895-x. [PubMed: 29690887]. [PubMed Central: PMC5926536].

5. Goncalves LM, Tsuge MLT, Borghi VS, Miranda FP, Sales APA, Lucchetti ALG, et al. Spirituality, religiosity, quality of life and mental health among pantaneiros: A study involving a vulnerable population in pantanal wetlands, Brazil. J Relig Health. 2018;57(6):2431-43. doi: 10.1007/s10943-018-0681-4. [PubMed:30066264]. 
6. Lima S, Teixeira L, Esteves R, Ribeiro F, Pereira F, Teixeira A, et al. Spirituality and quality of life in older adults: A path analysis model. BMC Geriatr. 2020;20(1):259. doi:10.1186/s12877-020-01646-0. [PubMed: 32727391]. [PubMed Central: PMC7391807].

7. Heidari M, Borujeni MG, Rafiei H. The assessment effect of spiritual care on hopelessness and depression in suicide attempts. $J$ Relig Health. 2019;58(4):1453-61. doi:10.1007/s10943-017-0473-2.[PubMed: 28932973].

8. Ellis MR, Thomlinson P, Gemmill C, Harris W. The spiritual needs and resources of hospitalized primary care patients. J Relig Health. 2013;52(4):1306-18. doi: 10.1007/s10943-012-9575-z. [PubMed: 22311475].

9. Ramondetta LM, Sun C, Surbone A, Olver I, Ripamonti C, Konishi T, et al. Surprising results regarding MASCC members' beliefs about spiritual care. Support Care Cancer. 2013;21(11):2991-8. doi: 10.1007/s00520013-1863-y. [PubMed: 23775154]. [PubMed Central: PMC3951207].

10. Best M, Butow P, Olver I. Do patients want doctors to talk about spirituality? A systematic literature review. Patient Educ Couns. 2015;98(11):1320-8. doi:10.1016/j.pec.2015.04.017.[PubMed: 26032908]

11. Cook CC. The faith of the psychiatrist. Ment Health Relig Cult. 2011;14(1):9-17. doi: 10.1080/13674671003622673.

12. Zakaria Kiaei M, Salehi A, Moosazadeh Nasrabadi A, Whitehead D, Azmal M, Kalhor R, et al. Spirituality and spiritual care in Iran: Nurses' perceptions and barriers. Int Nurs Rev. 2015;62(4):584-92. doi: 10.1111/inr.12222. [PubMed: 26572823].

13. Best M, Butow P, Olver I. Doctors discussing religion and spirituality: A systematic literature review. Palliat Med. 2016;30(4):327-37. doi: 10.1177/0269216315600912. [PubMed: 26269325].

14. Neathery M, Taylor EJ, He Z. Perceived barriers to providing spiritual care among psychiatric mental health nurses. Arch Psychiatr Nurs. 2020;34(6):572-9. doi: 10.1016/j.apnu.2020.10.004. [PubMed: 33280682].

15. Moosavi S, Rohani C, Borhani F, Akbari ME. Factors affecting spiritual care practices of oncology nurses: A qualitative study. Support Care Cancer. 2019;27(3):901-9. doi: 10.1007/s00520-018-4378-8. [PubMed: 30066199].

16. Musa AS, Al Qadire MI, Aljezawi M, Tawalbeh LI, Aloush S, Albanian FZ. Barriers to the provision of spiritual care by nurses for hospitalized patients in Jordan. Res Theory Nurs Pract. 2019;33(4):392-409. doi: 10.1891/1541-6577.33.4.392. [PubMed: 31666395].

17. Oxhandler HK, Giardina TD. Social workers' perceived barriers to and sources of support for integrating clients' religion and spirituality in practice. Soc Work. 2017;62(4):323-32. doi: 10.1093/sw/swx036. [PubMed: 28957580].

18. Best M, Butow P, Olver I. Why do we find It so hard to discuss spirituality? A qualitative exploration of attitudinal barriers. J Clin Med. 2016;5(9). doi: 10.3390/jcm5090077. [PubMed: 27598212]. [PubMed Central: PMC5039480].

19. Polit DF, Beck CT. Nursing research: Generating and assessing evidence for nursing practice. Wolters Kluwer; 2020. p. 486-510.

20. Graneheim UH, Lundman B. Qualitative content analysis in nursing research: concepts, procedures and measures to achieve trustworthiness. Nurse Educ Today. 2004;24(2):105-12. doi: 10.1016/j.nedt.2003.10.001. [PubMed: 14769454].

21. Kyngäs H, Kääriäinen M, Elo S. The Trustworthiness of Content Analysis. The Application of Content Analysis in Nursing Science Research. Springer International Publishing; 2020. p. 41-8.

22. Hvidt NC, Nielsen KT, Korup AK, Prinds C, Hansen DG, Viftrup DT, et al. What is spiritual care? Professional perspectives on the concept of spiritual care identified through group concept mapping. BMJ Open. 2020;10(12). e042142. doi: 10.1136/bmjopen-2020-042142. [PubMed: 33372078]. [PubMed Central: PMC7772306].

23. Holmes C. Stakeholder views on the role of spiritual care in Australian hospitals: An exploratory study. Health Policy. 2018;122(4):389-95. doi: 10.1016/j.healthpol.2018.02.003. [PubMed: 29478877].

24. Keleshteri ZM, Rohani C. Spiritual interventions in health care studies in Iran: A systematic review. J Spiritual Ment Health. 2019;22(4):302-29. doi: 10.1080/19349637.2019.1620667.

25. Jafari N, Loghmani A, Puchalski CM. Spirituality and health care in Iran: Time to reconsider. J Relig Health. 2014;53(6):1918-22. doi: 10.1007/s10943-014-9887-2. [PubMed: 24912827].

26. Delgado C. A discussion of the concept of spirituality. Nurs Sci Q. 2005;18(2):157-62. doi: 10.1177/0894318405274828. [PubMed: 15802748]

27. Memaryan N, Rassouli M, Mehrabi M. Spirituality concept by health professionals in Iran: A qualitative study. Evid Based Complement Alternat Med. 2016;2016:8913870. doi: 10.1155/2016/8913870. [PubMed 27493675]. [PubMed Central: PMC4967431].

28. Bar-Sela G, Schultz MJ, Elshamy K, Rassouli M, Ben-Arye E, Doumit $\mathrm{M}$, et al. Training for awareness of one's own spirituality: A key factor in overcoming barriers to the provision of spiritual care to advanced cancer patients by doctors and nurses. Palliat Sup port Care. 2019;17(3):345-52. doi: 10.1017/S147895151800055X. [PubMed 30187841].

29. Ellington L, Billitteri J, Reblin M, Clayton MF. Spiritual care communication in cancer patients. Semin Oncol Nurs. 2017;33(5):517-25. doi 10.1016/j.soncn.2017.09.002. [PubMed: 29107530].

30. Rogers M. Utilizing availability and vulnerability to operationalize spirituality. Practising spirituality: Reflections on meaning-making in personal and professional contexts. Palgrave Macmillan Ltd;2017.p.145-64.

31. Koren ME, Papadimitriou C. Spirituality of staff nurses: Application of modeling and role modeling theory. Holist Nurs Pract. 2013;27(1):37-44. doi: 10.1097/HNP.ob013e318276fc38. [PubMed: 23211387].

32. Balboni MJ, Sullivan A, Enzinger AC, Epstein-Peterson ZD, Tseng YD Mitchell C, et al. Nurse and physician barriers to spiritual care provision at the end of life. J Pain Symptom Manage. 2014;48(3):400-10. doi: 10.1016/j.jpainsymman.2013.09.020. [PubMed: 24480531]. [PubMed Central: PMC4569089].

33. Abdollahyar A, Baniasadi H, Doustmohammadi MM, Sheikhbardesiri H, Yarmohammadian MH. Attitudes of Iranian nurses toward spirituality and spiritual care. J Christ Nurs. 2019;36(1):E11-6. doi: 10.1097/CNJ.0000000000000581. [PubMed: 30531521].

34. Holyoke P, Stephenson B. Organization-level principles and practices to support spiritual care at the end of life: A qualitative study BMCPalliat Care.2017;16(1):24. doi:10.1186/s12904-017-0197-9. [PubMed: 28399827]. [PubMed Central: PMC5387203].

35. Narayanasamy A, Owens J. A critical incident study of nurses' responses to the spiritual needs of their patients. J Adv Nurs. 2001;33(4):446-55. doi: 10.1046/j.1365-2648.2001.01690.x. [PubMed: $11251732]$.

36. Rushton L. What are the barriers to spiritual care in a hospital setting? Br J Nurs. 2014;23(7):370-4. doi: 10.12968/bjon.2014.23.7.370. [PubMed: 24732989].

37. Wattis J, Rogers M, Ali G, Curran S. Bringing spirituality and wisdom into practice. Practice Wisdom. Brill; 2019. p. 155-68.

38. Brown O, Elkonin D, Naicker S. The use of religion and spirituality in psychotherapy: Enablers and barriers. J Relig Health. 2013;52(4):113146. doi: 10.1007/s10943-011-9551-z. [PubMed: 22076048].

39. Tao Z, Wu P, Luo A, Ho TL, Chen CY, Cheng SY. Perceptions and practices of spiritual care among hospice physicians and nurses in a Taiwanese tertiary hospital: a qualitative study. BMC Palliat Care. 2020;19(1):96. doi: 10.1186/s12904-020-00608-y. [PubMed: 32611340]. [PubMed Central: PMC7331138].

40. Rosmarin DH, Forester BP, Shassian DM, Webb CA, Bjorgvinsson $\mathrm{T}$. Interest in spiritually integrated psychotherapy among acute psychiatric patients. J Consult Clin Psychol. 2015;83(6):1149-53. doi: 10.1037/ccp0000046. [PubMed: 26280491]. [PubMed Central: PMC4658247].

41. Edwards A, Pang N, Shiu V, Chan C. The understanding of spirituality and the potential role of spiritual care in end-of-life and palliative care: a meta-study of qualitative research. Palliat Med.2010;24(8):75370. doi: 10.1177/0269216310375860. [PubMed: 20659977]. 